\title{
Electron localization by a donor in the vicinity of a basal stacking fault in GaN
}

\author{
P. Corfdir, ${ }^{1}$ P. Lefebvre, ${ }^{2,1}$ J. Ristić, ${ }^{1}$ J.-D. Ganière, ${ }^{1}$ and B. Deveaud-Plédran ${ }^{1}$ \\ ${ }^{1}$ Institut de Photonique et d'Electronique Quantiques, Ecole Polytechnique Fédérale de Lausanne (EPFL), 1015 Lausanne, Switzerland \\ ${ }^{2}$ Groupe d'Etude des Semiconducteurs, CNRS-Université Montpellier II, 34095 Montpellier, France \\ (Received 3 July 2009; revised manuscript received 18 September 2009; published 27 October 2009)
}

\begin{abstract}
We study the effects of the vicinity between a shallow donor nucleus and an $\mathrm{I}_{1}$-type basal stacking fault (BSF) in GaN. We propose a numerical calculation, in the "effective potential" formalism, of energies and envelope functions of electrons submitted to the conjunction of attractive potentials caused by the BSF and the donor. We show that the donor localizes the electron along the plane of the BSF, even when the donor lies as far as $10 \mathrm{~nm}$ from the BSF. Conversely, the presence of the BSF enhances the donor binding energy by up to a factor of 1.8, when the donor is placed exactly on the BSF. We briefly discuss the probability of occurrence of such a situation in, e.g., $a$-plane $\mathrm{GaN}$, as well as its consequences on transport and optical properties of this material.
\end{abstract}

DOI: 10.1103/PhysRevB.80.153309

PACS number(s): 71.55.Eq, 73.21.Fg, 73.22.Dj

The current interest in the growth of wurtzite GaN along the nonpolar [11-20] $a$-axis is fostered by the possibility of eliminating the internal electric fields that are induced in nitride-based quantum structures when grown along $[0001],{ }^{1-3}$ the polar $c$-axis. In such $a$-plane $\mathrm{GaN}$, however, large densities of a particular class of extended defects, namely, basal stacking faults (BSFs), are usually observed. After a number of theoretical and experimental studies, ${ }^{4-10}$ these BSFs are generally considered to consist in ultrathin (three monolayers), perfectly smooth insertions of cubiclike $\mathrm{GaN}$ in a wurtzite GaN matrix. Electrons are confined in the cubiclike layer, but with significant spreading of the wave function in the surrounding barrier. On the other hand, the corresponding valence-band discontinuity has been predicted to be such that the cubiclike layer constitutes a potential barrier for holes, leading for the overall structure to a type-II band line-up. ${ }^{7}$

In usual quantum wells (QWs) produced by epitaxy, potential fluctuations of diverse origins induce, especially at low temperatures, a natural in-plane localization of carriers (electrons, holes, or excitons). Quite generally, well-width fluctuations of typically one atomic monolayer occur during the epitaxial growth of QWs, leading to the localization of carriers where the QW is wider, i.e., with lower quantized energy. Moreover, even in nitride-based QWs with flat interfaces, the large effective mass of holes make them also sensitive to potential fluctuations due to alloy disorder in the well ${ }^{11}$ or in the barriers. ${ }^{12}$ In any case, the energy lost by, say, an exciton, when localized at such fluctuations, is larger than the energy that this exciton would lose by localizing onto a neutral donor in the bulk material. Donor-related excitonic transitions are consequently not observed in QWs: excitons are better localized by fluctuations than they are by donors. ${ }^{13}$

Given their intrinsic nature, BSFs represent the unprecedented situation of QWs without any well-width fluctuations. Consequently, and contrary to usual heterostructures produced by epitaxy, no in-plane exciton localization is expected. Now, GaN is a material that is quite generally produced with residual $n$-type doping classically induced by silicon or oxygen, both acting as shallow donors. Their binding energies are, respectively, of 30.4 and $33.2 \mathrm{meV}^{14}$ which is in very reasonable agreement with the result of an effectivemass approximation estimate: $28.8 \mathrm{meV} .^{15}$

In the present study, we discuss the effect of a donor atom on the energy of an electron confined in a BSF in wurtzite GaN. Conversely, we have also considered this situation as the effect of a BSF on the donor binding energy. By using a variational envelope-function calculation in the "effective potential" formalism, we demonstrate that the presence of a donor, less than $\sim 10 \mathrm{~nm}$ away from the BSF, essentially localizes the electron in the BSF plane. We calculate, in particular, the dependence of the in-plane extension and of the localization energy with the distance between the donor and the BSF.

The conduction-band profile along the [0001] direction $(z$ axis), perpendicular to the plane of an $\mathrm{I}_{1}$-type BSF in wurtzite GaN, is sketched in Fig. 1(a). The QW width is $L_{w}=1.5$, $\mathrm{c}_{0}=0.78 \mathrm{~nm}$, and the potential discontinuity, $V_{0}$, between the well (cubiclike $\mathrm{GaN}$ ) and the barriers (wurtzite $\mathrm{GaN}$ ) was taken equal to $270 \mathrm{meV}$, according to the existing literature. To calculate the subband extremum and the envelope function shown in Fig. 1(a), we have used an electron effective mass of $m_{e}=0.2 m_{0},{ }^{16}$ for both the well and barrier materials. Counted from the edge of the quantum well, we find a binding energy of the electron to the BSF equal to $46 \mathrm{meV}$.

Now, in order to account for the presence of both the BSF plane and the donor nucleus, and given the symmetry of the problem, we write the Hamiltonian of the electron in cylindrical coordinates,

$$
\begin{aligned}
H= & -\frac{\hbar^{2}}{2 m_{e}}\left(\frac{\partial^{2}}{\partial z^{2}}+\frac{1}{\rho^{2}} \frac{\partial^{2}}{\partial \theta^{2}}+\frac{1}{\rho} \frac{\partial}{\partial \rho}+\frac{\partial^{2}}{\partial \rho^{2}}\right)+V(z) \\
& -\frac{e^{2}}{4 \pi \varepsilon \sqrt{(z-d)^{2}+\rho^{2}}} .
\end{aligned}
$$

The potential profile $V(z)$ induced by the BSF is given by $V(z)=-V_{0}$, for $-L_{w} / 2<z<L_{w} / 2$, and $V(z)=0$, elsewhere. The position $(\rho=0, z=d)$ corresponds to the positively charged donor nucleus located at a distance $d$ from the BSF plane; $r=\sqrt{(z-d)^{2}+\rho^{2}}$ is the electron-nucleus distance and 


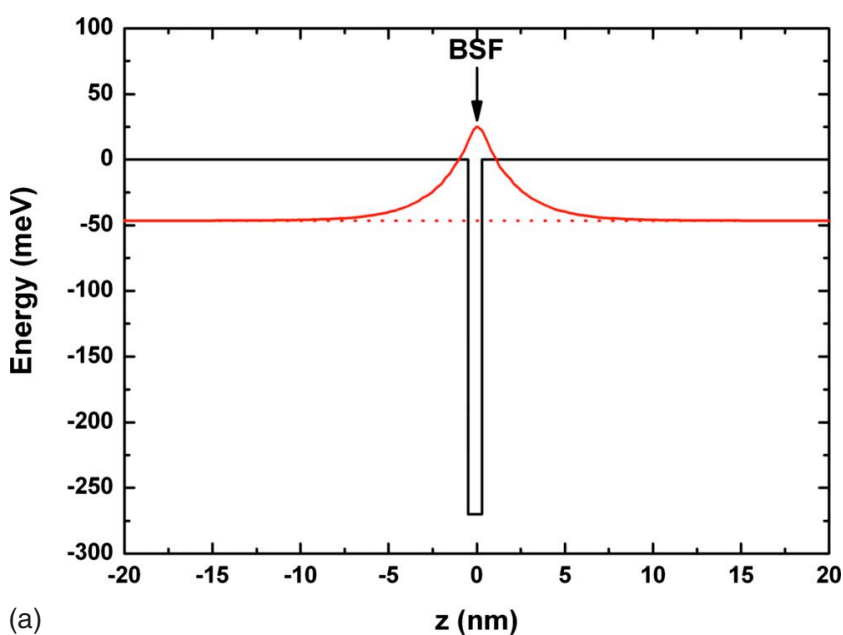

(a)

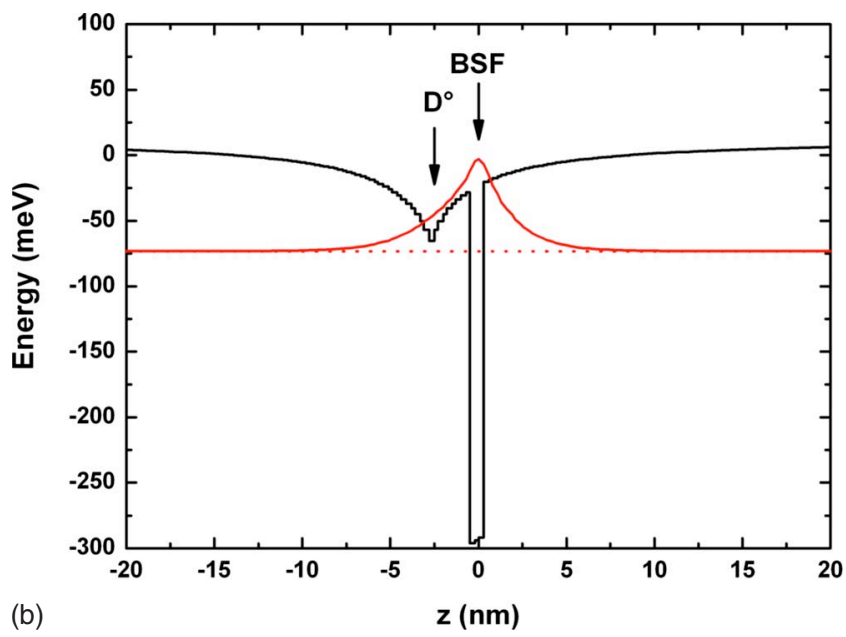

FIG. 1. (Color online) Band profiles, electron envelope functions (black and red curves, respectively) and energy (dotted red line) for an electron confined on a single BSF (a) and on a $\mathrm{D}^{\circ}$-BSF complex with distance $d$ of $2.5 \mathrm{~nm}$ (b).

the relative dielectric constant of $\mathrm{GaN} \varepsilon$ is taken equal to 9.5. ${ }^{17}$

We will now apply the so-called effective potential method, first developed by $\mathrm{Wu}$ for the problem of lowdimensional excitons. ${ }^{18}$ The versatility of this approach, based on the possibility to calculate quantized states of onedimensional quantum wells of arbitrary shape, was successfully applied to heterostructures with specific band alignments, ${ }^{19-22}$ and readily extended to the treatment of donor states in GaN QWs. ${ }^{23}$

According to this approach, in the context of the variational theorem, we use the following ansatz for the electron envelope function:

$$
\Psi_{\lambda}(\rho, z)=f(z) \varphi_{\lambda}(\rho)=f(z) \sqrt{\frac{2}{\pi}} \frac{e^{-\rho / \lambda}}{\lambda},
$$

where the $z$ dependence, described by the square-normalized function $f(z)$, is separated from the in-plane dependence $\varphi_{\lambda}(\rho)$. This latter term is a square-normalized twodimensional $1 s$ hydrogenic envelope function, with a charac- teristic in-plane extension parameter (pseudo-Bohr radius) $\lambda$, which we use as variational parameter. The aim being to minimize the expectation energy $\langle\Psi|H| \Psi\rangle_{\lambda}=E_{\lambda}$ against $\lambda$, thus yielding an approximation of the ground-state energy, it is convenient to average the envelope-function equation $H \Psi=E_{\lambda} \Psi$ over all possible in-plane positions,

$$
\begin{aligned}
\left\langle\varphi_{\lambda}|H| \Psi\right\rangle_{\lambda} & =E_{\lambda}\left\langle\varphi_{\lambda} \mid \Psi\right\rangle_{\lambda} \Leftrightarrow \int_{0}^{\infty} \varphi_{\lambda}^{*}(\rho) H \varphi_{\lambda}(\rho) f(z) 2 \pi \rho d \rho \\
& =E_{\lambda} f(z),
\end{aligned}
$$

which can be written as

$$
-\frac{\hbar^{2}}{2 m_{e}} \frac{d^{2} f(z)}{d z^{2}}+\tilde{V}_{\lambda}(z) f(z)=E_{\lambda} f(z) .
$$

The advantage of this method is that we are left with a onedimensional effective-mass equation that $f(z)$ has to obey. The usual potential term has now been replaced by the socalled effective potential $\widetilde{V}_{\lambda}(z)$ given by

$$
\begin{aligned}
\tilde{V}(z)= & \frac{2 \hbar^{2}}{m_{e}}\left(\int_{0}^{\infty} \frac{1}{\lambda^{3}} e^{-2 \rho / \lambda} d \rho-\int_{0}^{\infty} \frac{1}{\lambda^{4}} e^{-2 \rho / \lambda} \rho d \rho\right)+V(z) \\
& -\int_{0}^{\infty} \frac{e^{2}}{\pi \lambda^{2} \varepsilon \sqrt{(z-d)^{2}+\rho^{2}}} e^{-2 \rho / \lambda} \rho d \rho
\end{aligned}
$$

which reduces to

$$
\tilde{V}_{\lambda}(z)=\frac{\hbar^{2}}{2 m_{e} \lambda^{2}}+V(z)-\frac{e^{2}}{2 \pi \varepsilon \lambda} \int_{0}^{\infty} \frac{x e^{-x}}{\sqrt{x^{2}+\left(\frac{2(z-d)}{\lambda}\right)^{2}}} d x .
$$

The shape and details of this potential, shown in Fig. 1(b), were not a priori decided. They simply result, in a strictly mathematical way, from the ansatz that we made on the shape of the envelope function. The first and the last terms on the right-hand side of Eq. (6) correspond, respectively, to the kinetic energy of the electron and to the electrostatic interaction between the electron and the charged donor nucleus, averaged on all in-plane positions of the electron. For a given value of $\lambda$, one can determine the effective potential $\widetilde{V}_{\lambda}(z)$ [Fig. 1(b)] and then solve Eq. (4) by a finiteelement technique. We impose $f(z)=0$ at the boundaries of the system, cut the $z$ axis into thin slabs of constant potential that mimic the overall potential profile, and finally apply the Numerov method. (For more information on the method, we refer the reader, e.g., to Ref. 24.)

The resulting energy implicitly depends on the in-plane extension parameter, $\lambda$. According to the variational theorem, we now have to minimize the overall electron energy by optimization of $\lambda$, thus maximizing the overall "binding energy" of the donor-BSF system.

Figure 2 shows the spatial distribution in the $a$-plane of electron probability resulting from this calculation, for three different values of $d$. In all cases, the electron is found to be strongly localized along the BSF plane. The larger the distance $d$, the weaker the localization, but it is important to 

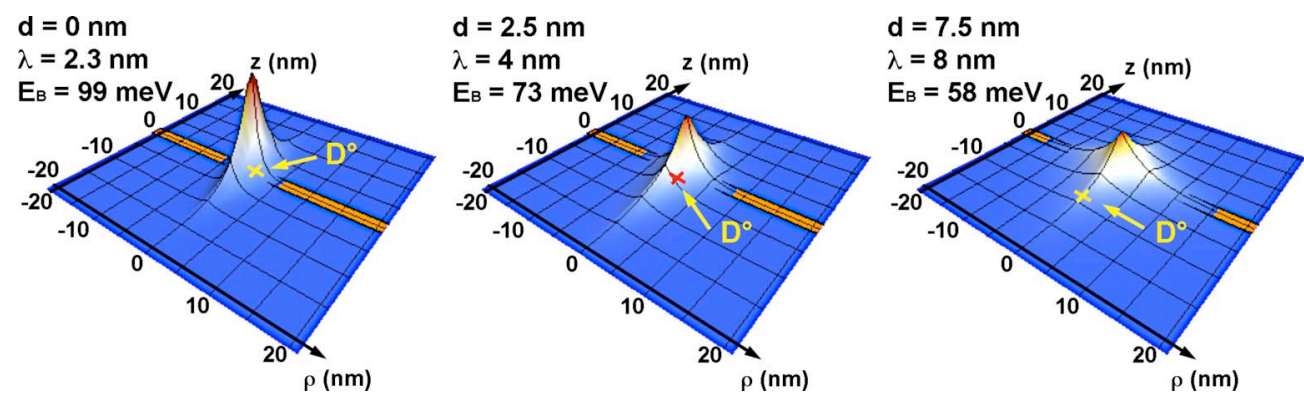

FIG. 2. (Color online) Spatial distribution in the $a$-plane (11-20) of the electron probability for donors located $0,2.5$ and $7.5 \mathrm{~nm}$ from the BSF plane. The orange rectangle and the cross represent the BSF plane and the donor nucleus, respectively.

remark that the effect of the donor nucleus remains significant, even when $d$ is as large as $10 \mathrm{~nm}$. In terms of binding energy (see Fig. 3), when $d$ tends to infinity, the minimum energy for the electron corresponds to its localization on the "bare" BSF, i.e., the above-mentioned $46 \mathrm{meV}$. When $d$ is reduced to zero, the binding energy monotonously increases up to $99 \mathrm{meV}$. The difference of $99-46=53 \mathrm{meV}$ is nothing but the binding energy of the donor increased by the confinement, i.e., 1.8 times the binding energy of the donor in bulk GaN. The discrepancy of this value from the binding energy of a two-dimensional donor ${ }^{25}$ comes naturally from the shallowness of the QW formed by the BSF that leads to a spreading of the electron wave function into the barriers. Conversely, this energy difference between the bare BSF $(-46 \mathrm{meV})$ and the $\mathrm{D}^{\circ}$-BSF complex could as well serve as a definition of the in-plane localization energy for an electron along the BSF plane. This localization energy therefore reaches a maximum of $53 \mathrm{meV}$, for $d=0$, and we note that it is still as large as $9 \mathrm{meV}$, for $d=10 \mathrm{~nm}$. In practical cases, given the statistical distribution of donor-BSF distances, we expect a statistical distribution of localization energies and of characteristic localization radii.

The typical density of residual donors in nonintentionally doped $\mathrm{GaN}$ is of $10^{17} \mathrm{~cm}^{-3}$, i.e., one donor every $\sim 20 \mathrm{~nm}$. The typical densities of BSFs in $a$-plane GaN vary between $10^{6} \mathrm{~cm}^{-1}$ in the so-called "bundle" regions and $10^{4} \mathrm{~cm}^{-1}$, in

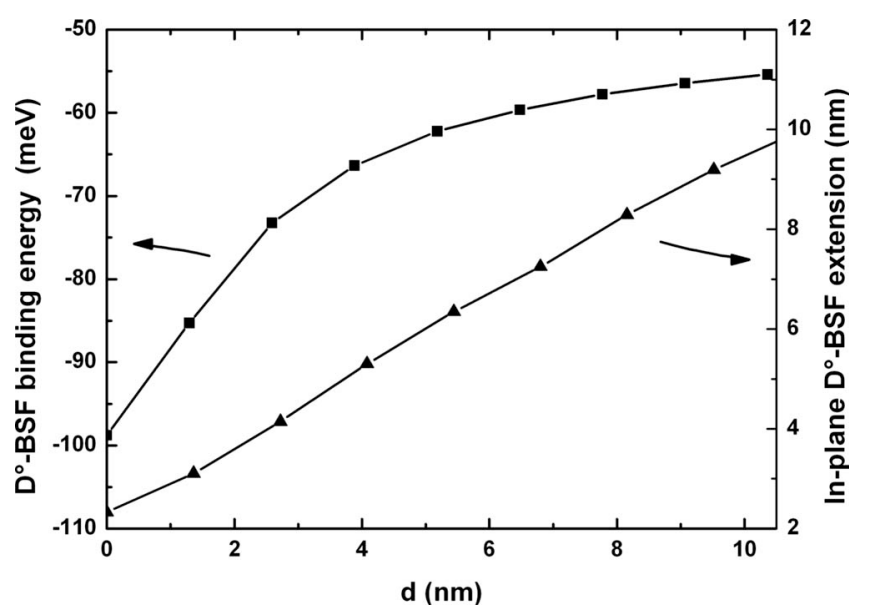

FIG. 3. Binding energy and in-plane extension (square and triangle symbols) of an electron bound to a $\mathrm{D}^{\circ}$-BSF in respect with $d$, the distance between the charged donor nucleus and the BSF plane. the "diluted" zones. ${ }^{9}$ Even if we consider that the presence of BSFs has no influence on the spatial positioning of donors, we see clearly that the situation addressed in the present paper is extremely frequent in state-of-the-art $a$-plane $\mathrm{GaN}$ :

(i) In bundles where the inter-BSF distance is of the order of $10 \mathrm{~nm}$, there exists no such things as a bare donor or a bare BSF, and only $\mathrm{D}^{\circ}$-BSF complexes can occur;

(ii) In diluted regions where the BSFs are separated by $1 \mu \mathrm{m}$, the volume fraction of material that is concerned by donor-BSF complexes is at least of $2 \%$.

In both cases, electrons localized in the surroundings of BSF planes may screen piezoelectric fields theoretically expected along the $c$ axis (perpendicular to the BSFs), ${ }^{26}$ but not yet convincingly observed by optical spectroscopy.

From these results, we can first conclude that, in case of high densities of such BSFs in GaN (as it is the case in state-of-the-art nonpolar GaN samples, where BSF densities as high as $10^{6} \mathrm{~cm}^{-1}$ are reported ${ }^{9}$ ), the conductivity properties of the material might be affected by an enhanced donor binding energy. Full experimental evidence of such an effect is still lacking, but we can mention, for example, the activation ratios of Si donors at $300 \mathrm{~K}$, which were measured to be comprised between 0.1 and 0.5 in nonpolar GaN layers, ${ }^{27}$ whereas this ratio is of 1 in $c$-plane GaN. Such an incomplete ionization of donors may result from an increased donor binding energy in the presence of BSFs.

We can also infer that the optical properties of BSF-bound excitons are most probably tributary to the statistical distribution of donors in the vicinity of the BSFs, necessarily leading to some in-plane localization of those excitons. Temperature-dependent measurements performed by Paskov et al. have, indeed, revealed some localization mechanism of excitons inside BSFs themselves, ${ }^{14}$ with a localization energy of $20 \mathrm{meV}$, close to the $17 \mathrm{meV}$ observed by our group. ${ }^{28}$ The emission energy of the nonlocalized BSF exciton is $3.433 \mathrm{eV}$, whereas the emission energy of the localized state is centered at $3.417 \mathrm{eV}$, with a full width at half maximum of $26 \mathrm{meV}$. In their original study, Paskov et al. excluded that this fairly large broadening could arise from large local strain variation and proposed instead that it may be caused by the fact that BSFs are gathered into bundles. ${ }^{14,29}$ However, we have recently shown by cathodoluminescence that the emission from isolated BSF is as broad as the one from bundles of BSFs [see Fig. 1(a) in Ref. 30]. As neither strain nor the inhomogeneous spatial distribution of BSFs can explain the broad emission line of BSF excitons, we 
propose donors to be the origin of the localization of excitons in BSFs. The statistical distribution of donor-BSF distances can then explain the broad emission line. Concerning the structure of these $\left(\mathrm{D}^{\circ}-\mathrm{BSF}\right) \mathrm{X}$ complexes, we can safely infer that the two electrons will be essentially located in the BSF plane, with some repulsive Coulomb interaction between them. The hole, attracted by the two electrons and repelled by both the BSF plane (type-II) and by the positive donor nucleus, should then be located in the wurtzite material surrounding the $\mathrm{BSF}$, at the opposite side from the donor nucleus. The ground state of this complex is just the initial state of the optical transition that is observed experimentally. The final state of this transition is nothing but the one that we calculate here: once one electron has recombined with the hole, we are, indeed, left with the $\mathrm{D}^{\circ}$-BSF system, in its ground state. To calculate the photon energy observed by photoluminescence, we should perform the full calculation of the initial state, which involves three mobile particles and multiple interactions. The energies of the initial and final states, as well as the recombination probability, will all exhibit individual dependencies on the donor-BSF distance, $d$. Such a calculation is complicated and outside the scope of the present paper. Finally, we want to point out that the formalism detailed in this paper can be directly extended to other classes of materials, such as $\mathrm{SiC}$, which also contain optically active BSFs. ${ }^{31}$

In summary, we have shown that the presence of donor nuclei in the vicinity of an $\mathrm{I}_{1}$-type BSF in wurtzite $\mathrm{GaN}$, leads to localization of electrons along the plane of the BSF. The localization energy increases monotonously when the donor-BSF distance is reduced, with a maximum of $53 \mathrm{meV}$ when the donor lies in the BSF. We also showed that the here modeled situation is very frequent in $\mathrm{GaN}$ samples containing substantial amounts of BSFs, like those samples grown along the $a$-plane, considering the typical residual doping of this material. In addition to the necessary effects of enhanced donor binding energy on $n$-type conductivity, we finally inferred that such donor-BSF complexes could be the origin of the localization of BSF-bound excitons that has been reported for $a$-plane $\mathrm{GaN}$.

We acknowledge financing by the Swiss National Science Foundation through Quantum Photonics NCCR and Project No. 119840.
${ }^{1}$ F. Bernardini and V. Fiorentini, Phys. Rev. B 57, R9427 (1998).

${ }^{2}$ M. Leroux, N. Grandjean, M. Laügt, J. Massies, B. Gil, P. Lefebvre, and P. Bigenwald, Phys. Rev. B 58, R13371 (1998).

${ }^{3}$ N. Akopian, G. Bahir, D. Gershoni, M. D. Craven, J. S. Speck, and S. P. DenBaars, Appl. Phys. Lett. 86, 202104 (2005).

${ }^{4}$ G. Salviati, M. Albrecht, C. Zanotti-Fregonara, N. Armani, M. Mayer, Y. Shreter, M. Guzzi, Yu. V. Melnik, K. Vassilevski, V. A. Dmitiev, and H. P. Strunk, Phys. Status Solidi A 171, 325 (1999).

${ }^{5}$ R. Liu, A. Bell, F. A. Ponce, C. Q. Chen, J. W. Yang, and M. A. Khan, Appl. Phys. Lett. 86, 021908 (2005).

${ }^{6}$ X. Ni, U. Özgür, Y. Fu, N. Biyikli, J. Xie, A. A. Baski, H. Morkoç, and Z. Liliental-Weber, Appl. Phys. Lett. 89, 262105 (2006).

${ }^{7}$ C. Stampfl and C. G. Van de Walle, Phys. Rev. B 57, R15052 (1998).

${ }^{8}$ Y. T. Rebane, Y. G. Shreter, and M. Albrecht, Phys. Status Solidi A 164, 141 (1997).

${ }^{9}$ T. Gühne, Z. Bougrioua, P. Venneguès, M. Leroux, and M. Albrecht, J. Appl. Phys. 101, 113101 (2007).

${ }^{10}$ Z. Z. Bandić, T. C. McGill, and Z. Ikonić, Phys. Rev. B 56, 3564 (1997).

${ }^{11}$ Y. Narukawa, Y. Kawakami, M. Funato, S. Fujita, S. Fujita, and S. Nakamura, Appl. Phys. Lett. 70, 981 (1997).

${ }^{12}$ M. Gallart, A. Morel, T. Talierco, P. Lefebvre, B. Gil, J. Allègre, H. Mathieu, N. Grandjean, M. Leroux, and J. Massies, Phys. Status Solidi A 180, 127 (2000).

${ }^{13}$ D. Bimberg, J. Christen, T. Fukunaga, H. Nakashima, D. E. Mars, and J. N. Miller, J. Vac. Sci. Technol. B 5, 1191 (1987).

${ }^{14}$ P. P. Paskov, B. Monemar, A. Toropov, J. P. Bergman, and A. Usui, Phys. Status Solidi C 4, 2601 (2007).

${ }^{15}$ K. Kornitzer, T. Ebner, K. Thonke, R. Sauer, C. Kirchner, V. Schwegler, M. Kamp, M. Leszczynski, I. Grzegory, and S. Po- rowski, Phys. Rev. B 60, 1471 (1999).

${ }^{16}$ I. Vurgaftman, J. R. Meyer, and L. R. Ram-Mohan, J. Appl. Phys. 89, 5815 (2001).

${ }^{17}$ P. Y. Yu and M. Cardona, Fundamentals of Semiconductors, 3rd ed. (Springer, New York, 2005).

${ }^{18}$ J.-W. Wu, Solid State Commun. 67, 911 (1988).

${ }^{19}$ E. L. Ivchenko, A. V. Kavokin, V. P. Kochereshko, G. R. Pozina, I. N. Uraltsev, D. R. Yakovlev, R. N. Bicknell-Tassius, A. Waag, and G. Landwehr, Phys. Rev. B 46, 7713 (1992).

${ }^{20}$ R. Zimmermann and D. Bimberg, Phys. Rev. B 47, 15789 (1993).

${ }^{21}$ A. Bellabchara, P. Lefebvre, P. Christol, and H. Mathieu, Phys. Rev. B 50, 11840 (1994).

${ }^{22}$ P. Bigenwald, P. Lefebvre, T. Bretagnon, and B. Gil, Phys. Status Solidi B 216, 371 (1999).

${ }^{23}$ A. Morel, P. Lefebvre, T. Taliercio, M. Gallart, B. Gil, and H. Mathieu, Mater. Sci. Eng., B 82, 221 (2001).

${ }^{24}$ A. Soba, Comm. Comp. Phys. 5, 914 (2009).

${ }^{25}$ G. Bastard, Phys. Rev. B 24, 4714 (1981).

${ }^{26}$ Y. J. Sun, O. Brandt, U. Jahn, T. Y. Liu, A. Trampert, S. Cronenberg, S. Dhar, and K. H. Ploog, J. Appl. Phys. 92, 5714 (2002).

${ }^{27}$ M. McLaurin, T. E. Mates, F. Wu, and J. S. Speck, J. Appl. Phys. 100, 063707 (2006)

${ }^{28}$ P. Corfdir, P. Lefebvre, J. Levrat, A. Dussaigne, J.-D. Ganière, D. Martin, J. Ristić, T. Zhu, N. Grandjean, and B. DeveaudPlédran, J. Appl. Phys. 105, 043102 (2009).

${ }^{29}$ P. P. Paskov, R. Schifano, B. Monemar, T. Paskova, S. Figge, and D. Hommel, J. Appl. Phys. 98, 093519 (2005).

${ }^{30}$ P. Corfdir, J. Ristić, P. Lefebvre, A. Dussaigne, D. Martin, J.-D. Ganière, N. Grandjean, and B. Deveaud-Plédran, Appl. Phys. Lett. 94, 201115 (2009).

${ }^{31}$ See for instance, J. Camassel and S. Juillaguet, Phys. Status Solidi B 245, 1337 (2008). 